\title{
Sleep Disturbances: Implications for Cannabis Use, Cannabis Use Cessation, and Cannabis Use Treatment
}

\author{
Kimberly A. Babson • Marcel O. Bonn-Miller
}

Published online: 18 March 2014

(C) Springer International Publishing AG (outside the USA) 2014

\begin{abstract}
Cannabis may be used, among certain individuals, for its actual and/or perceived sleep-promoting properties. Although evidence suggests that cannabis is likely beneficial for sleep initiation, over time individuals may develop tolerance to these benefits, leading to greater use in order to maintain the same sleep-inducing effects. This form of use likely contributes to the development of problematic cannabis use patterns, including cannabis use disorders. Evidence also points to sleep as an important consideration in terms of understanding cannabis withdrawal and relapse. Here, sleep disturbances have been reported as a primary symptom of withdrawal, with studies revealing that this increase in sleep disruption during discontinuation of cannabis use may be a significant risk factor for relapse. Therefore, it is likely important to consider interventions aimed at providing alternative
\end{abstract}

\footnotetext{
K. A. Babson

Center for Innovation to Implementation, VA Palo Alto Health Care System, 795 Willow Road (152-MPD), Menlo Park, CA 94025, USA

K. A. Babson ( $\square)$

Department of Psychiatry and Behavioral Sciences, Stanford School of Medicine, 401 Quarry Road, Stanford, CA 94305-5717, USA

e-mail: kbabson@stanford.edu

M. O. Bonn-Miller

Center of Excellence in Substance Abuse Treatment and Education, Philadelphia VA Medical Center, 3900 Woodland Avenue,

Philadelphia, PA 19104, USA

e-mail: marcel.bonn-miller@va.gov

M. O. Bonn-Miller

National Center for PTSD \& Center for Innovation to

Implementation, VA Palo Alto Health Care System, 795 Willow

Road (152-MPD), Menlo Park, CA 94025, USA

M. O. Bonn-Miller

Department of Psychiatry, Perelman School of Medicine, University of Pennsylvania, 3535 Market Street, Philadelphia, PA 19104, USA
}

means to cope with and/or treat sleep disturbances (e.g., behavioral or pharmacological approaches) as adjuncts to interventions for cannabis use disorders to improve treatment outcomes.

Keywords Cannabis $\cdot$ Sleep $\cdot$ Cannabis withdrawal $\cdot$ Cannabis treatment $\cdot$ Cannabis use disorders $\cdot$ Insomnia $\cdot$ Sleep disturbances

\section{Introduction}

Approximately 97 million Americans over the age of 12 years ( $31 \%$ of the overall population) have tried cannabis, with 4.3 million Americans (1.3\% of the population) meeting the criteria for problematic cannabis use (defined as hazardous use, abuse, or dependence) in 2009 [1]. On the basis of this prevalence pattern, it has been estimated that the need for cannabis use disorder (CUD) treatment will more than double by 2020 [2]. Contingency management, motivational enhancement, and cognitive behavioral interventions are currently the most efficacious evidence-based interventions offered for treatment of CUDs [3]. Despite the presence of such effective interventions, and patients' desire to quit, cannabis use cessation attempts are often met with high rates of lapse and relapse [4-6], indicating significant room for improvement in the treatment of problematic cannabis use.

Previous research has shown that $63 \%$ of adults engaged in cannabis use treatment (i.e., cognitive behavioral intervention and motivation enhancement) relapse (defined as a return to the previous level of use or abandonment of the abstinence goal) to regular use within 4 months, with the rates of relapse increasing to over $70 \%$ by 16 months [7]. In addition to full relapses, recent work has demonstrated that lapses (defined as a slip or violation of the abstinence goal) are also common. Moore and Budney [5] demonstrated that among outpatient 
adults engaged in treatment for a CUD, $24 \%$ lapsed by 1 month, $46 \%$ within 3 months, and $71 \%$ within 6 months. For this reason, it is important to identify malleable mechanisms implicated in poor cannabis use cessation outcomes to improve and generally refine existing treatments for CUD. Sleep disturbance is one factor which has shown recent theoretical and empirical promise in terms of understanding cannabis use and predicting cannabis use cessation outcomes. By understanding the role of sleep in regard to cannabis use, one may optimize interventions for individuals with CUD.

The purpose of this review is to highlight the existing literature pertaining to the comorbidity between cannabis use and sleep disturbances, including implications for future empirical work and treatments. We begin by discussing the neurobiological underpinnings of the association between sleep and cannabis, followed by a discussion of clinical work aimed at understanding how disturbed sleep may lead to cannabis use. Next, we discuss mechanisms by which cannabis use may lead to increased difficulties with sleep, including how sleep may affect cannabis use cessation outcomes.

\section{Neurobiology of Sleep and Cannabis Comorbidity}

Preclinical and clinical research demonstrates that cannabis alters the sleep-wake cycle [8]. Cannabis comprises a multitude of chemical compounds, with the primary psychoactive constituent being $\Delta^{9}$-tetrahydrocannabinol $\left(\Delta^{9}\right.$-THC), which has neural implications that are mediated through cannabinoid (i.e., CB1) receptors located in the brain [9]. One of the primary neural effects of $\Delta^{9}$-THC is sedation [10]. There are two primary mechanisms proposed to explain this impact. First, endogenous cannabinoids have been shown to increase the level of adenosine, a sleep-promoting agent [11]. Second, neurons in the lateral hypothalamus involved in regulation of arousal systems express CB1 receptors, resulting in inhibition of the arousal system [11]. Some studies have shown that immediate administration of $\Delta^{9}$-THC reduces sleep latency [12]. However, in studies using high-dose $\Delta^{9}$-THC, or among cannabis-naïve participants, $\Delta^{9}$-THC tends to have an activating response that has been associated with increased latency to sleep onset [13-15]. These contrasting findings could also be explained by work that has shown that $\Delta^{9}$-THC may have a biphasic influence whereby stimulating effects are first experienced, followed by sedating effects [16].

Cannabidiol (CBD) is a nonpsychoactive component of cannabis that can counter the excitatory effects, and potentiate the sedative effects, of $\Delta^{9}$-THC [8]. CBD administered immediately has been shown to perform as a short-acting hypnotic in rats [8]. Specifically, CBD administration has been shown to have sleep-inducing (with a $20 \mathrm{mg} / \mathrm{kg}$ dose) and sleepmaintenance (with a $40 \mathrm{mg} / \mathrm{kg}$ dose) qualities in rats, with tolerance to these effects developing after long-term administration [8]. However, among humans, administration of CBD (i.e., $15 \mathrm{mg}$ ) has been shown to have an alerting affect, which can counter the sedating qualities of $\Delta^{9}$-THC [17]. Despite the disparate findings regarding the individual effects of $\Delta^{9}$-THC and CBD, when combined, $\Delta^{9}-\mathrm{THC}$ and CBD appear to have an antagonistic effect on each other in terms of the sleep-wake cycle $[8,18]$. Together, the dose and the timing of administration appear to be important factors to consider when evaluating the impact of $\Delta^{9}$-THC and CBD.

Both cannabis and sleep disturbances have also been associated with reduction of activity in the same regions of the prefrontal cortex [19-22]. The prefrontal cortex has been shown to play a primary role in normal sleep, and reductions in activity in the prefrontal cortex have been observed among individuals with insomnia [21] or sleep deprivation [22], and among heavy cannabis users abstinent for 30 days [19]. The orbitofrontal cortex (OFC) has been a region of particular interest, as both discontinuation of cannabis use and insomnia are associated with decreased OFC glucose metabolism [21, 23], and $\Delta^{9}$-THC administration has been associated with increases in OFC glucose metabolism, which may function to decrease insomnia [24]. Taken together, these cortical disruptions offer a potential model from which to understand the association between sleep disturbance and cannabis use.

\section{Poor Sleep Can Contribute to Coping-Oriented Cannabis Use}

Understanding the reasons or motives for substance use is also helpful for grasping the nature of substance use behavior. Recent work has demonstrated that individuals, particularly those with psychological vulnerabilities, for example, posttraumatic stress disorder (PTSD), other anxiety disorders, and depression, may use cannabis for coping-related reasons [25-28]. Those reporting coping as a primary motive for cannabis use have been shown to be at higher risk of elevated frequency and amount of use, as well as the development of a CUD [29-32]. Following from this line of work, there has been a developing focus on understanding specific states or symptoms for which individuals use cannabis to cope.

Sleep disturbances have emerged as a set of symptoms for which individuals appear particularly apt to use cannabis for coping reasons [33••]. Research has suggested that administration of an oromucosal cannabis-based medicine extract (2.7 mg $\Delta^{9}$-THC, $2.5 \mathrm{mg}$ CBD) improves short-term sleep problems among individuals with insomnia and chronic pain [34]. This initial empirical work is further supported by neurobiological research demonstrating that the psychoactive components of cannabis, such as $\Delta^{9}$-THC, may facilitate the onset of sleep [34, 35]. However, over time, individuals may habituate or develop tolerance to the sleep-inducing and selfreported sleep-enhancing qualities of cannabis, thereby 
requiring more in order to obtain the same desired result [36]. Indeed, the beneficial effect of cannabis on self-reported sleep quality is less frequently noted among long-term cannabis users [36].

Clinical research has also provided evidence for the use of cannabis as a means to cope with sleep disturbances among adults and adolescents. For example, among adolescent cannabis users without a use disorder (aged 16-22 years), 69.6\% reported using it to sleep [37]. Lower rates have been observed among regular long-term cannabis users (i.e., those using cannabis for at least 10 years), of which $50 \%$ reported using cannabis to improve sleep [38]. Among medical cannabis patients, Bonn-Miller el al. [39] demonstrated that $48.1 \%$ use cannabis to aid with insomnia, whereas slightly higher rates have been observed among medical cannabis patients receiving cannabis for physical health reasons [40]. Indeed, of those receiving medical cannabis for treatment of pain, 83-85\% reported improved sleep, with higher rates of sleep benefit observed among those with mood disorders (93\%) [40].

The use of cannabis for sleep promotion has also been observed among nonmedical users with psychological disorders, with $69.4 \%$ of individuals with psychosis reporting cannabis use to improve sleep [41]. Among a sample of individuals with PTSD, Bonn-Miller et al. [42] demonstrated that, compared with women with PTSD who self-reported relatively good sleep, women with elevated symptoms of both PTSD and disturbed sleep were more likely to report using cannabis as a means to cope. A follow-up study among medical cannabis patients demonstrated that those with elevated symptoms of PTSD used medical cannabis for the purposes of improving sleep, and that this pattern was associated with a heightened frequency of cannabis use [33••].

Taken together, research on coping motives among individuals with and without psychological vulnerabilities suggests that cannabis may initially serve to promote sleep initiation among individuals with sleep difficulties. However, the continued use of cannabis for these reasons may actually lead to long-term deficits in sleep architecture and severer cannabis use patterns. What follows from this work is a cyclical model whereby cannabis is used to cope with initial sleep difficulties that may or may not be associated with a psychological disorder (e.g., PTSD). As cannabis is used to cope with sleep difficulties, individuals may find that they begin using more cannabis than initially intended, with tolerance developing over time. Such heightened cannabis use then likely leads to greater disturbances in sleep during periods of abstinence, which can then prompt a return to cannabis use.

\section{Sleep Disturbance as a Cannabis Withdrawal Symptom}

A breadth of laboratory-based studies have identified sleep disturbance as a salient symptom of cannabis withdrawal
[43-46]. Controlled studies have shown that sleep disturbances increase during periods of abstinence and remit during periods of cannabis use [43]. An outpatient study of cannabis use cessation demonstrated that sleep disturbance was elevated over the course of a 45-day abstinence period [44], and studies of inpatient non-treatment-seeking regular users have demonstrated that withdrawal from cannabis increases both self-reported and objectively measured (i.e., polysomnography) sleep disturbances. Resumption of cannabis use [43], and oral administration of both low ( $30 \mathrm{mg} /$ day) and high $\left(90 \mathrm{mg} /\right.$ day) doses of $\Delta^{9}$-THC have been shown to alleviate these sleep problems [46-48].

In contrast, one study demonstrated no effect of orally administered $\Delta^{9}$-THC on objective measures of sleep within the context of cannabis withdrawal [49], whereas in a second study, Haney et al. [50] demonstrated that orally administered $\Delta^{9}$-THC (60 mg/day) increased sleep onset latency during cannabis abstinence. However, in this same study, a combination of lofexidine and orally administered $\Delta^{9}$-THC resulted in significant improvements in both self-reported and objectively measured sleep during cannabis use cessation.

In a more recent study, Vandrey et al. [51 ••] specifically examined the effect of addressing poor sleep during cannabis withdrawal in a within-subject crossover study among 20 daily cannabis users. The participants completed alternating periods of cannabis use and abstinence in a controlled environment. During a single abstinence phase, a sleep medication (zolpidem) was administered, and a placebo pill was administered during a second abstinence period. This study demonstrated that during the placebo abstinence phase, the participants experienced reductions in sleep efficiency, sleep time, stage 1 and stage 2 sleep, REM latency, and self-reported sleep quality. The participants also demonstrated an increase in sleep onset latency and the amount of time spent in REM sleep compared with periods of cannabis use. Administration of zolpidem attenuated all sleep disturbances except increases in sleep onset latency.

In summary, outpatient and inpatient studies combined have identified sleep disturbance as a salient cannabis withdrawal symptom. Administration of cannabis (through either smoking or oral administration of $\Delta^{9}$-THC), sleep medication $[51 \bullet \cdot]$, and a combination of lofexidine and orally administered $\Delta^{9}$-THC have all been shown to attenuate withdrawalrelated sleep disturbance.

\section{Sleep Disturbance Increases the Risk of Cannabis Lapse/Relapse}

As sleep disturbance has been documented as a cannabis withdrawal symptom, it is not surprising that poor sleep has been shown to be one factor that is predictive of lapse/relapse to cannabis use $[44,45,52,53 \bullet \bullet, 54 \bullet \cdot]$. Retrospective studies 
first documented these associations. In one study, 67 daily cannabis users who had made a cannabis use cessation attempt in the previous 30 days were interviewed by phone regarding perceptions of withdrawal symptoms. Of this sample, $65 \%$ indicated that poor sleep was a primary symptom that led to their relapse [45]. Furthermore, in two studies of nontreatment-seeking cannabis users, 32-47\% reported poor sleep during a previous time of abstinence, and 48-77\% indicated that they had relapsed to cannabis use or increased the use of other substances in order to improve sleep quality $[55,56]$.

The role of sleep on cannabis use lapse/relapse has also been supported by laboratory-based experimental work. Haney [52] demonstrated that decreases in sleep disturbance resulted in decreased risk of lapse/relapse to cannabis use (as measured by a laboratory analog task). More recently, sleep disturbance among cannabisdependent veterans prior to a cannabis use cessation attempt was shown to be predictive of increased risk of lapse within the first 2 days of the cessation attempt [54••], and heightened cannabis use during the 6 months following the cessation attempt [53・•]. In contrast to these findings, studies have demonstrated that a number of medications shown to improve withdrawal-related sleep disturbances (i.e., lofexidine, mirtazapine, quetiapine) have not led to reductions in the risk of lapse/relapse to cannabis use in the laboratory model developed by Haney et al. [50, 57, 58].

\section{Clinical Implications}

A breadth of research has demonstrated the efficacy of both pharmacological (e.g., benzodiazepine hypnotics) and behavioral (cognitive behavioral therapy for insomnia, CBT-I) interventions for the treatment of insomnia and sleep disturbances [59-61]. Comparisons between pharmacotherapy and behavioral interventions have demonstrated comparable treatment effects, with pharmacotherapy offering a more immediate impact on sleep and behavioral interventions linked to greater long-term and sustained treatment gains [62-64]. Studies have demonstrated that the best sleep treatment outcomes are observed among individuals who are initially treated with a combination of pharmacotherapy and behavioral interventions and then continue treatment only with the behavioral intervention [64]. Although treatment effectiveness exists for both forms of intervention, it is important to note that no study has examined comparative efficacy or effectiveness of these interventions among individuals with a history of substance use disorders. This limitation of existing work is likely due to the fact that pharmacotherapy for insomnia is typically not recommended for individuals with a history of substance use disorders owing to the risk of the development of dependence [61]. For this reason, additional research among substance use populations is needed.

Although the findings of recent work have converged to demonstrate that sleep disturbances impact substance use and quit success, this research is still in its infancy, particularly in relation to the evaluation of sleep interventions for substance use outcomes. Although the impact of CBT-I or sleep medications on cannabis use cessation has yet to be thoroughly evaluated, research drawing from the treatment of alcohol use disorders provides some insight. Research has shown that among individuals recovering from alcohol dependence, behavioral interventions (e.g., CBT-I) improve self-reported and objective measures of sleep immediately after the intervention $[65,66]$ and at 3 and 6 months after the intervention [65]. However, there has yet to be any documentation of an effect of behavioral sleep interventions on alcohol use cessation outcomes (e.g., abstinence, relapse) [65, 66].

Despite the extensive research support for pharmacotherapy and behavioral interventions for insomnia, widespread dissemination and integration of these interventions into specialty care treatment centers (e.g., substance abuse treatment) has been limited. For this reason, additional research is needed to understand the potential impact of administering a sleep intervention prior to, or within the context of, CUD treatment.

\section{Conclusions}

Although there remains a general dearth of literature on the associations between sleep disturbance, cannabis use, and cannabis use cessation, existing work points to a cyclical relation whereby cannabis use is maintained, and likely initiated, by sleep disturbances stemming both from symptoms of existing psychopathological disorders and periods of cannabis abstinence. Therefore, interventions that provide an alternative method to cope with disturbed sleep may not only decrease the perceived need to use cannabis to cope with sleep problems, but may also potentially lessen the salience of sleep-related cannabis withdrawal, decreasing the risk of lapse/relapse. Although studies of sleep interventions among alcohol users provide some evidence for the utility of cognitive behavioral therapy for co-occurring sleep disturbances, clinical trials examining the impact of such interventions on substance use outcomes are lacking. Future work would benefit from the investigation of the impact of behavioral and pharmacological sleep interventions, either as standalone interventions or as an adjunct to other interventions, for individuals with co-occurring sleep disturbance and cannabis use. 


\section{Compliance with Ethics Guidelines}

Conflict of Interest Kimberly A. Babson and Marcel O. Bonn-Miller declare they have no conflict of interest.

Human and Animal Rights and Informed Consent All procedures followed were in accordance with the ethical standards of the responsible committee on human experimentation (institutional and national) and with the Helsinki Declaration of 1975, as revised in 2008. Informed consent was obtained from all patients for their inclusion in the study.

\section{References}

Papers of particular interest, published recently, have been highlighted as:

•- Of major importance

1. Substance Abuse and Mental Health Services Administration. Results from the 2010 National Survey on Drug Use and Health: summary of national findings, NSDUH series H-41. Rockville: Substance Abuse and Mental Health Services Administration; 2011.

2. Gfroerer J, Epstein J. Marijuana initiates and their impact on future drug abuse treatment need. Drug Alcohol Depend. 1999;54:229-37.

3. Roffman R, Stephens R. Cannabis dependence: its nature, consequences and treatment. Cambridge: Cambridge University Press; 2006.

4. Budney A, Radonovich K, Higgins S, Wong C. Adults seeking treatment for marijuana dependence: a comparison with cocainedependent treatment seekers. Exp Clin Psychopharmacol. 1998;6: 419-26.

5. Moore B, Budney A. Relapse in outpatient treatment for marijuana dependence. J Subst Abuse Treat. 2003;25:85-9.

6. Stephens R, Roffman R, Simpson E. Adult marijuana users seeking treatment. J Consult Clin Psychol. 1993;61:1100-4.

7. Marijuana Treatment Project Research Group. Brief treatments for cannabis dependence: findings from a randomized multisite trial. J Consult Clin Psychol. 2004;72:455-66.

8. Monti J. Hypnoticlike effects of cannabidiol in the rat. Psychopharmacology. 1977;55:263-5.

9. Bolla K, Lesage S, Gamalso C, Neubauer D, Funderburk F, Cadet J, et al. Sleep disturbance in heavy marijuana users. Sleep. 2008;31: 901-8.

10. Fusar-Poli P, Crippa J, Bhattacharyya S, Borgwardt S, Allen P, Martin-Santos $\mathrm{R}$, et al. Distinct effect of $\Delta 9$-tetrahydrocannabinol and cannabidiol on neural activation during emotional processing. Arch Gen Psychiatry. 2009;66:95-105.

11. Murillo-Rodriguez E, Blanco-Centurion C, Sanchez C, Piomello D, Shiromani P. Anandamide enhances extracellular levels of adenosine and induces sleep: an in vivo microdialysis study. Sleep. 2003;26:943-7.

12. Cousens K, DiMascio A. Delta 9 THC as an hypnotic. An experimental study of three dose levels. Psychopharmacologia. 1973;33: 355-64.

13. Tassinari C, Ambrosetto G, Peraita-Adrado MR, Gastaut H. The neuropsychiatric syndrome of delta-9-tetrahydrocannabinol and cannabis intoxication in naïve subjects: a clinical and polygraphic study during wakefulness and sleep. In: Braude M, Szara S, editors. Pharmacology of marijuana. New York: Raven; 1976. p. 357-75.

14. Gillin J, Kotin J, Post R. Sleep during one week of administration of delta-9-tetrahydrocanabinol to psychiatric patients. J Sleep Res. 1972;1:44.
15. Bobon D, Schulz H, Mattke D, Simonova O. Influence of synthetic8-tetrahydrocannabinol on all-night sleep EEG in man. In: Jovanovic U, editor. The nature of sleep. Stuttgart: Fischer; 1973.

16. Weisz D, Gunnell D, Teyler T, Vardaris R. Changes in hippocampal CA1 population spikes following administration of delat-9-THC. Brain Res Bull. 1982;8:155-62.

17. Nicholson A, Turner C, Stone B, Robson P. Effect of $\Delta-9$ tetrahydrocannabinol and cannabidiol on nocturnal sleep and early-morning behavior in young adults. J Clin Psychopharmacol. 2004;24:305-13.

18. Moreton J, Davis W. Electroencephalographic study of the effects of tetrahydrocannabinols on sleep in the rat. Neuropharmacology. 1973;12:897-907.

19. Bolla K, Brown K, Eldreth D, Tate K, Cadet J. Dose-related neurocognitive effects of marijuana use. Neurology. 2002;59: 1337-43.

20. Chuah Y, Venlatraman V, Dinges D, Chee M. The neural basis of interindividual variability in inhibitory efficiency after sleep deprivation. J Neurosci. 2006;26:7156-62.

21. Drummond S, Smith M, Orff H, Chengazi V, Perlis M. Functional imaging of the sleeping brain: review of findings and implications for the study of insomnia. Sleep Med Rev. 2004;8:227-42.

22. Muzur A, Pace-Schott E, Hobson J. The prefrontal cortex in sleep. Trends Cogn Sci. 2002;6:475-81.

23. Eldreth D, Matochik J, Cadet J, Bolla K. Abnormal brain activity in prefrontal brain regions in abstinent marijuana users. Neuroimaging. 2004;23:914-20.

24. Volkow N, Gillespie H, Mullani N, Tancredi L, Grant C, Valentine $\mathrm{A}$, et al. Brain glucose metabolism in chronic marijuana users at baseline and during marijuana intoxication. Psychiatry Res. 1996;67:29-38.

25. Boden MT, Babson KA, Vujanovic AA, Short NA, Bonn-Miller MO. Posttraumatic stress disorder and cannabis use characteristics among military veterans with cannabis dependence. Am J Addict. 2013;22:277-84.

26. Bonn-Miller MO, Vujanovic AA, Feldner MT, Bernstein A, Zvolensky MJ. Posttraumatic stress symptom severity predicts marijuana use coping motives among traumatic event-exposed marijuana users. J Trauma Stress. 2007;20:577-86.

27. Buckner JD, Schmidt NB, Bobadilla L, Taylor J. Social anxiety and problematic cannabis use: evaluating the moderating role of stress reactivity and perceived coping. Behav Res Ther. 2006;44:1007-15.

28. Passie T, Emrich HM, Karst M, Brandt SD, Halpern JH. Mitigation of post-traumatic stress symptoms by cannabis resin: a review of the clinical and neurobiological evidence. Drug Test Anal. 2012;4: 649-59.

29. Cooper M. Motivations for alcohol use among adolescents: Development and validation of a four-factor model. Psychol Assess. 1994;6:117-28.

30. Fox C, Towe S, Stephens R, Walker D, Roffman R. Motives for cannabis use in high-risk adolescent users. Psychol Addict Behav. 2011;25:492-500.

31. Hides L, Lubman D, Cosgrave E, Buckby J, Killackey E, Yung A. Motives for substance use among young people seeking mental health treatment. Early Interv Psychiatr. 2008;2:188-94.

32. Johnson K, Mullin J, Marshall E, Bonn-Miller M, Zvolensky M. Exploring the mediational role of coping motives for marijuana use in terms of the relation between anxiety sensitivity and marijuana dependence. Am J Addict. 2010;19:277-82.

33.• Bonn-Miller MO, Babson KA, Vandrey R. Using cannabis to help you sleep: Heightened frequency of medical cannabis use among those with PTSD. Drug Alcohol Depend. 2014;136:162-5. Improvement in sleep is the primary motivation for medical cannabis use among medical cannabis users with elevated symptoms of PTSD. 
34. Russo E, Guy G, Robson P. Cannabis, pain, and sleep: lessons from therapeutic clinical trials of Sativex, a cannabis-based medicine. Chem Biodivers. 2007;4:1729-43.

35. Schierenbeck T, Riemann D, Berger M, Homyak M. Effect of illicit recreational drugs upon sleep: cocaine, ecstasy and marijuana. Sleep Med Rev. 2008;12:381-9.

36. Halikas J, Weller R, Morse C, Hoffmann R. A longitudinal study of marijuana effects. Int J Addict. 1985;20:701-11.

37. Boys A, Marsden J, Strang J. Understanding reasons for drug use amongst young people: a functional perspective. Health Educ Res. 2001;16:457-69.

38. Reilly D, Didcott P, Swift W, Hall W. Long-term cannabis use: characteristics of users in an Australian rural area. Addiction. 1998;93:837-46.

39. Bonn-Miller MO, Boden MT, Bucossi MM, Babson KA. Selfreported cannabis use characteristics, patterns, and helpfulness among medical cannabis users. Am J Drug Alcohol Abuse. 2014;40:23-30.

40. Walsh Z, Callaway R, Belle-Isle L, Capler R, Kay R, Lucas P, et al. Cannabis for therapeutic purposes: patient characteristics, access, and reasons for use. Int J Drug Pol. 2013;24:511-6.

41. Schaub M, Fanghaenel K, Stohler R. Reasons for cannabis use: Patients with schizophrenia versus matched healthy controls. Aust N Z J Psychiatr. 2008;42:1060-5.

42. Bonn-Miller MO, Babson KA, Vujanovic AA, Feldner MT. Sleep problems and PTSD symptoms interact to predict marijuana use coping motives: a preliminary investigation. J Dual Diagn. 2010;6:111-22.

43. Budney A, Hughes J, Moore B, Novy P. Marijuana abstinence effects in marijuana smokers maintained in their home environment. Arch Gen Psychiatry. 2001;58:917-24.

44. Budney A, Moore B, Vandrey R, Hughes J. The time course and significance of cannabis withdrawal. J Abnorm Psychol. 2003;112: 393-402.

45. Budney A, Vandrey R, Hughes J, Thostenson J, Bursac Z. Comparison of cannabis and tobacco withdrawal syndrome. Am J Psychiatry. 2008;161:1967-77.

46. Budney A, Vandrey R, Hughes J, Moore B, Bahrenburg B. Oral delta-9-tetrahydrocannabinol suppresses cannabis withdrawal symptoms. Drug Alcohol Depend. 2007;86:22-9.

47. Haney M, Ward A, Comer S, Foltin R, Fischman M. Abstinence symptoms following oral THC administration to humans. Psychopharmacology. 1999;141:385-94.

48. Hart C, Ward A, Haney M, Comer S, Foltin R, Fischman M. Comparison of smoked marijuana and oral $\Delta^{9}$-tetrahydrocannabinol in humans. Psychopharmacology. 2002;164:407-15.

49. Haney M, Hart C, Vosburg S, Nasser J, Bennett A, Zubaran C, et al. Marijuana withdrawal in humans: effects of oral THC or divalproex. Neuropsychopharmacology. 2004;29:58-70.

50. Haney M, Hart C, Vosburg S, Comer S, Reed S, Foltin R. Effects of THC and lofexidine in a human laboratory model of marijuana withdrawal and relapse. Psychopharmacology. 2008;197:157-68.

51.• Vandrey R, Smith M, McCann U, Budney A, Curran E. Sleep disturbance and the effects of extended release zolpidem during cannabis withdrawal. Drug Alcohol Depend. 2011;117:38-44. Cannabis use cessation increases sleep disturbances, whereas intervention to address sleep problems attenuates this finding. Sleep interventions may be a beneficial addition to CUD treatment.

52. Haney M. Self-administration of cocaine, cannabis, and heroin in the human laboratory: benefits and pitfalls. Addict Biol. 2009;14:921.

53.• Babson K, Boden M, Bonn-Miller M. The impact of perceived sleep quality and sleep efficiency/duration on cannabis use during a self-guided quit attempt. Addict Behav. 2013;38:2707-13. Veterans with poor sleep quality prior to a quit attempt have less of a reduction in cannabis use during a quit attempt compared with those with relatively better sleep.

54.• Babson KA, Boden MT, Harris AHS, Stickle TR, Bonn-Miller MO. Poor sleep quality as a risk factor for lapse following a cannabis quit attempt. J Subst Abuse Treat. 2013;44:438-43. Veterans with poor sleep prior to a cannabis quit attempt are at greater risk of lapse/ relapse to cannabis use within the first 2 days of the quit attempt.

55. Copersino M, Boyd S, Tashkin D, Huestis M, Heishman S, Dermans J, et al. Cannabis withdrawal among non treatmentseeking adult cannabis user. Am J Addict. 2006;15:8-14.

56. Levin K, Copersino M, Heishman S, Liu F, Kelly D, Boggs D, et al. Cannabis withdrawal symptoms in non-treatment-seeking adult cannabis smokers. Drug Alcohol Depend. 2010;111:120-7.

57. Haney M, Hart C, Vosburg S, Comer S, Reed S, Cooper Z, et al. Effects of baclofen and mirtazapine on a laboratory model of marijuana withdrawal and relapse. Psychopharmacology. 2010;211:233-44.

58. Cooper Z, Foltin R, Hart C, Vosburg S, Comer S, Haney M. A human laboratory study investigating the effects of quetiapine on marijuana withdrawal and relapse in daily marijuana smokers. Addict Biol. 2013;18:993-1002.

59. Morgenthaler T, Kraemer M, Alessi C, Friedman L, Boehlecke B, Brown T, et al. Practice parameters for the psychological and behavioral treatment of insomnia: an update. An AASM report. Sleep. 2006;29:1415-9.

60. Morin C, Culbert J, Schwartz S. Nonpharmacological interventions for insomnia: a meta-analysis of treatment efficacy. Am J Psychiatry. 1994;151:1172-80.

61. Roehrs $T$, Roth $T$. Insomnia pharmacotherapy. Neurotherapeutics. 2012;9:728-38.

62. Morin C, Colecchi C, Stone J, Sood R, Brink D. Behavioral and pharmacological therapies for late-life insomnia: a randomized controlled trial. JAMA. 1999;28:991-9.

63. McClusky H, Milby J, Switzer P, William V, Wooten V. Efficacy of behavioral versus triazolam treatment in persistent sleep-onset insomnia. Am J Psychiatry. 1991;148:121-6.

64. Morin C, Vallieres A, Guay B, Ivers H, Savard J, Merette C, et al. Cognitive behavioral therapy, singly and combined with medication, for persistent insomnia. A randomized controlled trial. JAMA. 2009;301:2005-15.

65. Currie S, Clark S, Hodgins D, El-Guebaly N. Randomized controlled trial of brief cognitive-behavioural interventions for insomnia in recovering alcoholics. Addiction. 2004;99:1121-32.

66. Arnedt J, Conroy D, Rutt J, Aloia M, Brower K, Armitage R. An open trial of cognitive-behavioral treatment for insomnia comorbid with alcohol dependence. Sleep Med. 2007;8:176-80. 